Bulletin of the Section of Logic

Volume 47/2 (2018), pp. 89-106

http://dx.doi.org/10.18778/0138-0680.47.2.02

A. V. Figallo, Nora Oliva and Alicia Ziliani

\title{
FREE MODAL PSEUDOCOMPLEMENTED DE MORGAN ALGEBRAS
}

\begin{abstract}
Modal pseudocomplemented De Morgan algebras (or $m p M$-algebras) were investigated in A. V. Figallo, N. Oliva, A. Ziliani, Modal pseudocomplemented De Morgan algebras, Acta Univ. Palacki. Olomuc., Fac. rer. nat., Mathematica 53,1 (2014), pp. 65-79, and they constitute a proper subvariety of the variety of pseudocomplemented De Morgan algebras satisfying $x \wedge(\sim x)^{*}=\left(\sim\left(x \wedge(\sim x)^{*}\right)\right)^{*}$ studied by H. Sankappanavar in 1987. In this paper the study of these algebras is continued. More precisely, new characterizations of $m p M$-congruences are shown. In particular, one of them is determined by taking into account an implication operation which is defined on these algebras as weak implication. In addition, the finite $m p M$-algebras were considered and a factorization theorem of them is given. Finally, the structure of the free finitely generated $m p M$-algebras is obtained and a formula to compute its cardinal number in terms of the number of the free generators is established. For characterization of the finitely-generated free De Morgan algebras, free Boole-De Morgan algebras and free De Morgan quasilattices see: $[16,17,18]$.
\end{abstract}

Keywords: Pseudocomplemented De Morgan algebras, congruences, free algebras.

\section{Introduction}

In 1949, P. Ribenboim ([19]) showed that the class of distributive $p$-algebras, whose study began V. Glivenko in [9], constitutes a variety. More precisely, he proved that distributive $p$-algebras are bounded distributive lattices with 
an additional unary operation * which satisfies the following identities: (R1) $x \wedge(x \wedge y)^{*}=x \wedge y^{*},(\mathrm{R} 2) x \wedge 0^{*}=x$ and (R3) $0^{* *}=0$.

A particular case of distributive $p$-algebras are pseudocomplemented De Morgan algebras which A. Romanowska ([20]) called $p M$-algebras. Recall that an algebra $\left\langle L, \wedge, \vee, \sim,{ }^{*}, 0,1\right\rangle$ of type $(2,2,1,1,0,0)$ is called a $p M$ algebra if $\langle L, \wedge, \vee, \sim, 0,1\rangle$ is a De Morgan algebra ([12]) (see also [1, 3]) and $\left\langle L, \wedge, \vee,{ }^{*}, 0,1\right\rangle$ is a distributive $p$-algebra. Let us observe that in this definition there are not any relationship between the operators $\sim$ and *

In 1978, A. Monteiro introduced tetravalent modal algebras as a generalization of three-valued Łukasiewicz algebras ([3]) by omitting the identity $\nabla(x \wedge y)=\nabla x \wedge \nabla y$ and they have been studied by different authors (see $[5,6,8,13,14])$. On the other hand, in order to find the maximal subclass of pseudocomplemented De Morgan algebras which admit a structure of tetravalent modal algebra, in [6] the subvariety of $p M$-algebras which satisfies: (tm) $x \vee \sim x \leq x \vee x^{*}$ was introduced and they called them modal pseudocomplemented De Morgan algebras (or $m p M$-algebras).

Besides, it is worth mentioning that this new class of algebras constitutes a proper subvariety of the variety $\mathcal{V}_{0}$ of all pseudocomplemented De Morgan algebras satisfying the identity: $x \wedge(\sim x)^{*}=\left(\sim\left(x \wedge(\sim x)^{*}\right)\right)^{*}$, studied by H. Sankappanavar in [22].

Furthermore, in [7] we described a topological duality for $m p M$-algebras and we characterized the congruences on these algebras by means of special subsets of their associated space. Taking into account these results we obtained the subdirectly irreducible $m p M$-algebras, we determined the principal congruences and we showed that $m p M$-algebras constitute a discriminator variety. Moreover, we described the ternary and the dual ternary discriminator polynomials.

In order to continue the study of $m p M$-algebras, in this paper our aim is focus on determining the free modal pseudocomplemented De Morgan algebras. Here is a summary of our main results. In section 1, we briefly describe the results needed throughout this article. In section 2, we characterize the congruences on these algebras in two different ways. One of them by considering the notion of $\triangle$-filter and the other by means of the deductive systems associated with a new implication operation which we called weak implication. In section 3 , we study the finite $m p M$-algebras and the main result is Theorem 3.5 which shows a factorization of these algebras. In section 4 , which is the core of this paper we determine the free 
$m p M$-algebras with a finite set of free generators and show a formula to obtain its cardinal number in terms of the numbers of its generators. Finally, these results allow us to check again that the varieties of $m p M$-algebras and tetravalent modal algebras do not coincide.

\section{Preliminaries}

We refer the reader to the bibliography listed here as $[2,4,12]$ for specific details of the many basic notions and results of universal algebra including distributive lattices, De Morgan algebras and distributive $p$-algebras considered in this paper. On the other hand, we will take into account some of the results of $m p M$-algebras obtained in [7] which will be usefull in what follows. More precisely, recall that:

A modal pseudocomplemented De Morgan algebra (or $m p M$-algebra) is a pseudocomplemented De Morgan algebra $\left\langle A, \wedge, \vee, \sim,{ }^{*}, 0,1\right\rangle$ which satisfies:

(TM) $x \vee \sim x \leq x \vee x^{*}$, where $a \leq b$ if and only if $a=a \wedge b$.

In what follows we will denote these algebras by its underlying set and we will indicate with $\boldsymbol{m p} \boldsymbol{M}$ the variety of $m p M$-algebras.

Now, we will summarize the most important properties of these algebras which we need throughout this work.

(TM1) Let $A \in \boldsymbol{m} \boldsymbol{p} \boldsymbol{M}$. Then it hold:
(m1) $\quad x \wedge x^{*}=x^{* *} \wedge x^{*}=0$,
$(m 2)$
$x \wedge y=0$ iff $x \leq y^{*}$,
(m3) $\quad x \leq x^{* *}$,
$(m 4) \quad x^{* * *}=x^{*}$,
$(m 5) \quad x \wedge y=0$ iff $x^{* *} \wedge y=0$,
$(m 6)$
$x \leq y$ implies $y^{*} \leq x^{*}$,
$(m 7) \quad(x \vee y)^{*}=x^{*} \wedge y^{*}$,
$(x \wedge y)^{* *}=x^{* *} \wedge y^{* *}$,
$(m 9)$
$\left(x^{* *} \vee y^{* *}\right)^{* *}=(x \vee y)^{* *}$,
$(m 10)$
$\left(x \vee x^{*}\right)^{*}=0$,
$(m 11) \quad x \wedge(x \wedge y)^{*}=x \wedge y^{*}$,
$(m 12)$
$\sim x^{*} \vee \sim x=1$,
$(m 13) \quad(\sim x)^{*} \leq \sim x^{*}$,
$(m 14)$
$x \wedge \sim(\sim x)^{*} \leq x \wedge \sim x$,
$(m 15) \quad(\sim x)^{*} \wedge x \wedge \sim(\sim x)^{*}=0$,
(m16)
$\sim(\sim x)^{*} \vee \sim x \vee(\sim x)^{*}=1$,
(m17) $\quad\left(\sim(\sim x)^{*}\right)^{*} \leq x$,
(m18)
$(\sim(x \wedge y))^{*}=(\sim x)^{*} \wedge(\sim y)^{*}$,
$(m 19) \quad\left(x^{*} \wedge y\right)^{* *}=x^{*} \wedge y^{* *}$
$(m 20) \quad x^{*} \wedge y^{* *}=\left(x \vee y^{*}\right)^{*}$. 
(TM2) The subdirectly irreducible $m p M$-algebras are, up to isomorphism, the algebras described below $([7])$ :

(i) $A_{1}=\{0,1\}$, where $0<1, \sim 0=0^{*}=1, \sim 1=1^{*}=0$,

(ii) $A_{2}=\{0, c, 1\}$, where $0<c<1, \sim c=c, c^{*}=0, \sim 0=0^{*}=1$, $\sim 1=1^{*}=0$

(iii) $A_{3}=\{0, a, b, 1\}$ where $a \not \leq b, b \not \leq a$ and $0<a, b<1, \sim b=a^{*}=$ $b, \sim a=b^{*}=a, \sim 0=0^{*}=1, \sim 1=1^{*}=0$.

(TM3) $\boldsymbol{m} \boldsymbol{p} \boldsymbol{M}$ is locally finite, semisimple, residually small and residually finite ([7, Theorem 4.3]).

\section{Congruences. $\triangle$-filters and weak deductive systems}

Our next task is to describe the congruence lattice of $m p M$-algebras by means of certain subsets of the algebra differently to the one given in [7]. In order to do so, we need some previous notions.

Let $A$ be an $m p M$-algebra. The operations $\sim$ and * allow us to introduce a new unary operator $\triangle$ by means of the formula: $\triangle x=(\sim x)^{*} \wedge x$.

LEMma 2.1. Let $A \in \boldsymbol{m} \boldsymbol{p} \boldsymbol{M}$. Then the following properties are satisfied:

(T1) $\triangle 0=0, \triangle 1=1$,

(T3) $x \leq y$ implies $\triangle x \leq \triangle y$,

(T5) $(\sim \triangle x)^{*}=\triangle x$,

(T7) $\triangle \triangle x=\triangle x$,

(T9) $\triangle \sim \triangle x=\sim \triangle x$,

(T11) $x \in \triangle A$ iff $x=\triangle x$,

(T13) $\sim x \wedge \triangle x=0$,

(T15) $\triangle(\triangle x \vee y)=\triangle x \vee \triangle y$,
(T2) $\triangle x \leq x$,

(T4) $\sim \triangle x$ is the Boolean complement of $\triangle x$,

(T6) $\quad(\sim \triangle x)^{*}=\triangle(\sim x)^{*}$,

(T8) $(\triangle x)^{*}=\sim \triangle x$,

(T10) $\triangle(x \wedge y)=\triangle x \wedge \triangle y$,

(T12) $\triangle A$ is a subalgebra of $A$,

(T14) $x \vee \sim \triangle x=1$,

(T16) $\triangle(\sim \triangle x \vee y)=\sim \triangle x \vee \triangle y$.

Proof: We will only prove that (T4), (T5), (T7), (T10) and (T15) hold.

(T4) $\triangle x \wedge \sim \triangle x=(\sim x)^{*} \wedge x \wedge \sim\left((\sim x)^{*} \wedge x\right)=(\sim x)^{*} \wedge x \wedge(\sim(\sim$ $\left.x)^{*} \vee \sim x\right)=\left((\sim x)^{*} \wedge x \wedge \sim(\sim x)^{*}\right) \vee\left((\sim x)^{*} \wedge x \wedge \sim x\right)$ and so, by $(\mathrm{m} 15)$ and $(\mathrm{m} 1)$ we have that $\triangle x \wedge \sim \triangle x=0$. Furthermore, $\sim \triangle x \vee \triangle x=\sim$ $(\triangle x \wedge \sim \triangle x)=\sim 0=1$. 
(T5) By virtue of (T4), we have that $\sim \triangle x \wedge \triangle x=0$. On the other hand, let $k \in A$ be such that $k \wedge \sim \triangle x=0$. Hence, $\triangle x=(\triangle x \vee k) \wedge(\triangle x \vee \sim$ $\triangle x)=\triangle x \vee k$. Therefore, $k \leq \triangle x$ which completes the proof.

(T7) $\triangle \triangle x=\left(\sim\left((\sim x)^{*} \wedge x\right)\right)^{*} \wedge(\sim x)^{*} \wedge x=\left(\sim(\sim x)^{*} \vee \sim x\right)^{*} \wedge(\sim$ $x)^{*} \wedge x=\left(\sim(\sim x)^{*}\right)^{*} \wedge(\sim x)^{*} \wedge x$. Then, by (m17), (T6) and (T5) we have that $\triangle \triangle x=\left(\sim(\sim x)^{*}\right)^{*} \wedge(\sim x)^{*}=\triangle(\sim x)^{*}=\triangle x$.

(T10) From (T5), (m7), (T2) and (m6) it follows that $\triangle x \wedge \triangle y=(\sim$ $\triangle x)^{*} \wedge(\sim \triangle y)^{*}=(\sim \triangle x \vee \sim \triangle y)^{*}=(\sim(\triangle x \wedge \triangle y))^{*} \leq(\sim(x \wedge y))^{*}$. Hence, $\triangle x \wedge \triangle y \leq(\sim(x \wedge y))^{*} \wedge(x \wedge y)=\triangle(x \wedge y)$. The other inequality follows immediately.

(T15) From (T4) we have that $x=x \vee 0=x \vee(\triangle y \wedge \sim \triangle y)=$ $(x \vee \triangle y) \wedge(x \vee \sim \triangle y)$. From this statement and (T10) it follows (1) $\triangle x=\triangle(x \vee \triangle y) \wedge \triangle(x \vee \sim \triangle y)$. On the other hand, from (T2) we conclude that $\triangle x \vee \sim \triangle y \leq x \vee \sim \triangle y$ and so, by (T12) and (T11) we have that $\triangle x \vee \sim \triangle y \leq \triangle(x \vee \sim \triangle y)$. Hence, $(\triangle x \vee \sim \triangle y) \wedge \triangle(x \vee \triangle y) \leq \triangle(x \vee \sim$ $\triangle y) \wedge \triangle(x \vee \triangle y)$ and by (1) we infer that $(\triangle x \vee \sim \triangle y) \wedge \triangle(x \vee \triangle y) \leq \triangle x$. Therefore, $(\triangle x \vee \sim \triangle y) \wedge \triangle(x \vee \triangle y) \vee \triangle y \leq \triangle x \vee \triangle y$ and so, we get that $\triangle(x \vee \triangle y) \leq \triangle x \vee \triangle y$. Besides, it is simple to verify that $\triangle x \vee \triangle y \leq$ $\triangle(x \vee \triangle y)$.

Our next task is to determine the $m p M$-congruences taking into account the operator $\triangle$ introduced above. For this purpose we will start by defining the notion of $\triangle$-filter.

Definition 2.2. A filter $F$ of an mpM-algebra $A$ is a $\triangle$-filter of $A$, if it satisfies that the hypothesis $x \in F$ imply $\triangle x \in F$.

We will denote by $\mathcal{F}(A)$ the set of all $\triangle$-filters of $A$.

As a direct consequence of Definition 2.2 it follows that $\{1\}$ and $A$ are $\triangle$-filters of $A$. Furthermore, the intersection of a non-empty family of $\triangle$-filters of $A$ is a $\triangle$-filter of $A$.

From now on, if $H$ is a non-empty subset of $A$, we will denote by $F(H)$ and $F_{\triangle}(H)$ the filter and the $\triangle$-filter of $A$ generated by $H$ respectively. If $H=\{a\}$, we will write $F(a)$ and $F_{\triangle}(a)$ instead of $F(\{a\})$ and $F_{\triangle}(\{a\})$ respectively and they are called the principal filter and the principal $\triangle$ filter generated by $a$.

Proposition 2.3. Let $A \in \boldsymbol{m} \boldsymbol{p} \boldsymbol{M}, H \subseteq A$ and $a \in A$. Then it hold: 
(i) $F_{\triangle}(H)=F(\triangle H)$, where $F(X)$ is the filter generated by $X \subseteq A$,

(ii) $F_{\triangle}(a)=F(\triangle a)$,

(iii) $F_{\triangle}(H \cup\{a\})=F(H \cup\{\triangle a\})$, if $H$ is $a \triangle$-filter of $A$.

Proof: (i) $F(\triangle H)$ is a $\triangle$-filter of $A$. Indeed, if $x \in F(\triangle H)$, then there exists $\triangle h_{1}, \triangle h_{2}, \ldots, \triangle h_{n} \in \triangle(H)$ such that $\triangle h_{1} \wedge \triangle h_{2} \wedge, \ldots, \wedge \triangle h_{n} \leq x$ and so by (T3), (T10) and (T7) we have that $\triangle h_{1} \wedge \triangle h_{2} \wedge, \ldots, \wedge \triangle h_{n} \leq$ $\triangle x$. Therefore, $\triangle x \in F(\triangle H)$. Besides, from (T2) it follows that $H \subseteq$ $F(\triangle H)$. On the other hand, if $T$ is a $\triangle$-filter of $A$ such that $H \subseteq T$, then $\triangle H \subseteq T$ and taking into account that $T$ is a filter we have that $F(\triangle H) \subseteq T$, hence the proof is completed.

(ii) It is a direct consequence of (i) by considering $H=\{a\}$.

(iii) It is routine.

From now on, we will denote by $\operatorname{Con}(A)$ the congruence lattice of $A$ and by $A / R$ the quotient algebra of $A$ by $R$. Besides, for $x \in A$ the equivalence class of $x$ modulo $R$ will be denoted by $|x|_{R}$.

In Theorem 2.4 the relationship between the congruences and the $\triangle$-filters of an $m p M$-algebra is determined.

Theorem 2.4. Let $A \in \boldsymbol{m} \boldsymbol{p} \boldsymbol{M}$. Then the following statements hold:

(i) $\operatorname{Con}(A)=\{R(F): F \in \mathcal{F}(A)\}$, where $R(F)=\{(x, y) \in A \times A$ : there exists $f \in F$ such that $x \wedge f=y \wedge f\}$.

(ii) The lattices $C o n(A)$ and $\mathcal{F}(A)$ are isomorphic considering the applications $\Theta \longmapsto F_{\Theta}$ and $F \longmapsto R(F)$, which are inverse to one another.

Proof: (i) Firstly, we will show that $R(F) \in \operatorname{Con}(A)$. In order to do this we will only prove that $R(F)$ is compatible with $\sim$ and ${ }^{*}$. Let $(x, y) \in R(F)$. Then, there is $f \in F$ such that (1) $x \wedge f=y \wedge f$. Thus, (2) $\triangle f \in F$ and so, we have that $(\sim x \vee \sim f) \wedge \triangle f=(\sim y \vee \sim f) \wedge \triangle f$. From this last assertion and (T13) we get that $\sim x \wedge \triangle f=\sim y \wedge \triangle f$. Hence, by (2) we obtain that $(\sim x, \sim y) \in R(F)$. On the other hand, by (1) we have that $(x \wedge f)^{*}=(y \wedge f)^{*}$ and so, $f \wedge(x \wedge f)^{*}=f \wedge(y \wedge f)^{*}$. From this statement and $\mathrm{A} 6$ we infer that $f \wedge x^{*}=f \wedge y^{*}$. Hence, $\left(x^{*}, y^{*}\right) \in R(F)$. Furthermore, it is straightforward that $|1|_{R(F)}=F$. 
On the other hand, if $\Theta \in \operatorname{Con}(A)$ then $F_{\Theta}=|1|_{\Theta}$ is a $\triangle$-filter of $A$. Indeed, let $x \in|1|_{\Theta}$. Hence, $\left((\sim x)^{*}, 1\right) \in \Theta$ and so, $\left((\sim x)^{*} \wedge x, 1\right) \in \Theta$. This last statement implies that $\triangle x \in|1|_{\Theta}$.

Suppose now that $(x, y) \in R\left(|1|_{\Theta}\right)$. Then, there exists $f \in|1|_{\Theta}$ such that $x \wedge f=y \wedge f$. Since $(f, 1) \in \Theta$, we have that $(x \wedge f, x) \in \Theta$ and $(y \wedge f, y) \in \Theta$. Hence, $(x, y) \in \Theta$ and so, $R\left(|1|_{\Theta}\right) \subseteq \Theta$. The other inclusion is a consequence of the fact that $(x, y) \in \Theta$ implies that $f=\triangle((\sim \triangle x \vee y) \wedge(\sim$ $\triangle y \vee x) \wedge(\sim \triangle(\sim x \vee \sim y) \vee \sim x) \wedge(\sim \triangle(\sim x \vee \sim y) \vee \sim y)) \in|1|_{\Theta}$ and $x \wedge f=y \wedge f$.

(ii) It is routine.

It is worth mentioning that Theorem 2.4 allows us to obtain a new characterization of simple $m p M$-algebras as Proposition 2.5 shows.

Proposition 2.5. Let $A \in \boldsymbol{m p M}$. Then the following conditions are equivalent:

(i) $\triangle A=\{0,1\}$,

(ii) $A$ is a simple algebra.

Proof: (i) $\Rightarrow$ (ii) Suppose that there is a proper $\triangle$-filter $F$ of $A$ and let $x \in F, x \neq 1$. Since $\triangle x \in F \cap \triangle A$, from the hypothesis we have that $\triangle x=1$ or $\triangle x=0$. Hence, we infer that $x=1$ or $F=A$, but both of them are contradictions.

(ii) $\Rightarrow$ (i) Let $x \in A$ be such that $0<x<1$. Then, by Proposition 2.3 we have that $F(\triangle x)$ is a proper $\triangle$-filter of $A$ and so, by Theorem 2.4 we conclude that $A$ is not a simple $m p M$-algebra which is a contradiction.

From now until the end of this section, our attention is focused on giving another characterization of the $m p M$-congruences. In order to do so, we define an implication operation on them, which we called weak implication, as follows:

$$
x \mapsto y=\sim \triangle x \vee y .
$$

Proposition 2.6. The weak implication $\mapsto$ satisfies the following properties: 
(W1) $1 \mapsto x=x$,

(W2) $\quad x \mapsto 1=1$,

(W3) $\quad x \mapsto x=1$,

(W4) $\quad x \mapsto(y \mapsto x)=1$,

(W5) $\quad x \mapsto(y \mapsto z)=y \mapsto(x \mapsto z)$,

(W6) $\quad x \mapsto(y \mapsto z)=(x \mapsto y) \mapsto(x \mapsto z)$,

(W7) $\quad x \leq y$ implies $z \mapsto x \leq z \mapsto y$,

(W8) $\quad x \leq y$ implies $x \mapsto y=1$,

(W9) $\quad((x \mapsto y) \mapsto x) \mapsto x=1$,

(W10) $\quad x \mapsto(x \mapsto y)=x \mapsto y$,

(W11) $y \leq x \mapsto y$,

(W12) $\quad x \leq y$ implies $y \mapsto z \leq x \mapsto z$,

$(\mathrm{W} 13) \quad(x \vee y) \mapsto z \leq(x \mapsto z) \wedge(y \mapsto z)$,

$(\mathrm{W} 14) \quad x \mapsto(y \vee z)=(x \mapsto y) \vee(x \mapsto z)$,

$(\mathrm{W} 15) \quad x \mapsto(y \mapsto(x \wedge y))=1$,

(W16) $\quad x \mapsto(x \wedge y)=x \mapsto y$,

(W17) $\triangle x \wedge(x \mapsto y)=\triangle x \wedge y$,

(W18) $\quad x \mapsto \triangle x=1$.

Proof: We will only prove (W6), (W8), (W9), (W13) and (W15).

(W6) From (T16) and (T4) we have that $(x \mapsto y) \mapsto(x \mapsto z)=\sim$ $\triangle(\sim \triangle x \vee y) \vee \sim \triangle x \vee z=\sim(\sim \triangle x \vee \triangle y) \vee \sim \triangle x \vee z=(\triangle x \wedge \sim \triangle y) \vee \sim$ $\triangle x \vee z=\sim \triangle x \vee \sim \triangle y \vee z=x \mapsto(y \mapsto z)$

(W8) From the hypothesis and (T3) we obtain that $\sim \triangle y \leq \sim \triangle x$. Hence, $\sim \triangle y \vee y \leq \sim \triangle x \vee y$ and so, by (T14) we conclude the proof.

(W9) Observe that $(x \mapsto y) \mapsto x=\sim\left(\sim\left(\sim(\sim x)^{*} \vee \sim x \vee y\right)\right)^{*} \vee((\sim$ $\left.\left.x)^{*} \wedge x \wedge \sim y\right) \vee x=\sim\left(\sim\left(\sim(\sim x)^{*} \vee \sim x \vee y\right)\right)^{*} \vee x=\sim\left((\sim x)^{*} \wedge x \wedge \sim y\right)\right)^{*} \vee x$. On the other hand, by (m6) and (m3) it follows that $\sim\left((\sim x)^{*} \wedge x \wedge \sim\right.$ $y)^{*} \leq \sim(\sim x)^{* *} \leq x$. Hence, $\sim\left((\sim x)^{*} \wedge x \wedge \sim y\right)^{*} \leq x$ which implies that $(x \mapsto y) \mapsto x=x$ and so, by (W3) the proof is completed.

(W13) By (T3) we have that $\triangle x, \triangle y \leq \triangle(x \vee y)$ and so, $\sim \triangle(x \vee y) \vee z \leq$ $\sim \triangle x \vee z \mathrm{y} \sim \triangle(x \vee y) \vee z \leq \sim \triangle y \vee z$. Hence, $\sim \triangle(x \vee y) \vee z \leq(\sim \triangle x \vee z) \wedge(\sim$ 
$\triangle y \vee z)$ and therefore, $(x \vee y) \mapsto z \leq(x \mapsto z) \wedge(y \mapsto z)$.

(W15) $x \mapsto(y \mapsto(x \wedge y))=\sim \triangle x \vee \sim \triangle y \vee(x \wedge y)=\sim(\triangle x \wedge$ $\triangle y) \vee(x \wedge y)$. Hence, taking into account (T10) and (T14) we get that $x \mapsto(y \mapsto(x \wedge y))=\sim \triangle(x \wedge y) \vee(x \wedge y)=1$.

Definition 2.7. A subset $D$ of an mpM-algebra $A$ is a weak deductive system of $A$ (w.d.s.), if it satisfies that $1 \in D$ and the hyphotesis $x \in D$ and $x \mapsto y \in D$ imply $y \in D$.

We will denote by $\mathcal{D}(A)$ the set of all w.d.s. of $A$.

In Proposition 2.8 the relationship between the notions of $\triangle$-filters and weak deductive systems in $m p M$-algebras is determined.

Proposition 2.8. Let $A \in \boldsymbol{m} \boldsymbol{p} \boldsymbol{M}$ and $D \subseteq A$. Then the following conditions are equivalent:

(i) $D$ is a $\triangle$-filter of $A$,

(ii) $D$ is a w.d.s. of $A$.

Proof: (i) $\Rightarrow$ (ii) Let $x, x \mapsto y \in D$. Then, from the hypothesis we have that $\triangle x, \triangle x \wedge(x \mapsto y) \in D$. Hence, by (W17) we deduce that $\triangle x \wedge y \in D$ and taking into account that $\triangle x \wedge y \leq y$, we conclude that $y \in D$.

(ii) $\Rightarrow$ (i) Let $x, y \in D$. Then from (W15) we have that $x \mapsto(y \mapsto$ $(x \wedge y))=1 \in D$. Thus, from the hypothesis we infer that $x \wedge y \in D$. On the other hand, if $x, y \in A$ are such that $x \in D$ and $x \leq y$, then by (W8) we obtain that $x \mapsto y \in D$ and so, $y \in D$. Besides, if $x \in D$ by (W18) we conclude that $\triangle x \in D$.

Proposition 2.9 will play an important role in order to obtain the desired characterization of the $m p M$-congruences by means of weak deductive systems.

Proposition 2.9. Let $A \in \boldsymbol{m} \boldsymbol{p} \boldsymbol{M}$ and let $F$ be $a \triangle$-filter of $A$. Then the following conditions are equivalent:

(i) there exists $f \in F$ such that $x \wedge f=y \wedge f$,

(ii) $x \mapsto y \in F, y \mapsto x \in F, \sim(x \wedge y) \mapsto \sim y \in F, \sim(x \wedge y) \mapsto \sim x \in F$.

Proof: (i) $\Rightarrow$ (ii): From the hypothesis, (W16) and Proposición 2.8 we have that $x \mapsto(x \wedge f)=x \mapsto(y \wedge f) \in F$. Furthermore, by (W7) it follows 
that $x \mapsto(y \wedge f) \leq x \mapsto y$. Hence, from these assertions we conclude that $x \mapsto y \in F$. Similarly we have that $y \mapsto x \in F$.

On the other hand, from the hypothesis we infer that $(\sim(x \wedge y) \vee \sim$ $f) \wedge \triangle f=(\sim y \vee \sim f) \wedge \triangle f$. Hence, $((\sim(x \wedge y) \wedge \triangle f) \vee(\sim f \wedge \triangle f)) \wedge(\sim$ $f)^{*}=((\sim y \wedge \triangle f) \vee(\sim f \wedge \triangle f)) \wedge(\sim f)^{*}$ and so, by (m1) it follows that $\sim(x \wedge y) \wedge \triangle f \wedge(\sim f)^{*}=\sim y \wedge \Delta f \wedge(\sim f)^{*}$. Thus, taking into account that $\triangle f \leq(\sim f)^{*}$ we have that $\sim(x \wedge y) \wedge \triangle f=\sim y \wedge \triangle f$. From this last assertion and (W16) we obtain that $\sim(x \wedge y) \mapsto(\sim y \wedge \triangle f)=\sim$ $(x \wedge y) \mapsto(\sim(x \wedge y) \wedge \triangle f)=\sim(x \wedge y) \mapsto \triangle f$. Then, bearing in mind that $\triangle f \in F$ and that $\sim(x \wedge y) \mapsto(\sim y \wedge \triangle f) \leq \sim(x \wedge y) \mapsto \sim y$ we conclude that $\sim(x \wedge y) \mapsto \sim y \in F$. A similar reasoning allows us to assert that $\sim(x \wedge y) \mapsto \sim x \in F$ and so, the proof is completed.

(ii) $\Rightarrow$ (i): From the hypothesis and taking into account that $F$ is a $\triangle$-filter of $A$ we have that $f=\triangle((x \mapsto y) \wedge(y \mapsto x) \wedge(\sim(x \wedge y) \mapsto \sim$ $x) \wedge(\sim(x \wedge y) \mapsto \sim y)) \in F$. Furthermore, it holds that $x \wedge f=y \wedge f$.

As a direct consequence of Proposition 2.8, Proposition 2.9 and Theorem 2.4 we have Theorem 2.10.

Theorem 2.10. Let $A \in \boldsymbol{m} \boldsymbol{p} \boldsymbol{M}$. Then the following statements hold:

(i) $\operatorname{Con}(A)=\{R(D): D \in \mathcal{D}(A)\}$, where $R(D)=\{(x, y) \in A \times A$ : $x \mapsto y \in D, y \mapsto x \in D, \sim(x \wedge y) \mapsto \sim y \in D, \sim(x \wedge y) \mapsto \sim x \in D\}$.

(ii) The lattices $\operatorname{Con}(A)$ and $\mathcal{D}(A)$ are isomorphic considering the applications $\Theta \longmapsto D_{\Theta}$ and $D \longmapsto R(D)$, which are inverse to one another.

\section{Finite $m p M$-algebras}

The algebras which is of our concern now, are finite $m p M$-algebras. The main result of this section is Theorem 3.5 which provides us a factorization of these algebras. On the other hand, taking into account Proposition 2.3 we obtain Lemma 3.1 and Lemma 3.2 which are fundamental tools to prove Theorem 3.5.

Lemma 3.1. Let $A$ be a finite $m p M$-algebra and $F \subseteq A$. Then the following conditions are equivalent:

(i) $F$ is a $\triangle$-filter of $A$,

(ii) there is $a \in A$ such that $F=F(\triangle a)$. 
Proof: (i) $\Rightarrow$ (ii): From the hypothesis and taking into account that $A$ is a finite algebra, there is $a \in A$ such that $F=F(a)$. Hence, $\triangle a \in F$. From these assertions and (T2) we conclude that $\triangle a=a$. Therefore, $F=F(\triangle a)$.

(ii) $\Rightarrow$ (i): It is a direct consequence of Proposition 2.3.

Let $A \in \boldsymbol{m} \boldsymbol{p} \boldsymbol{M}$. A maximal $\triangle$-filter of $A$ is a $\triangle$-filter which is maximal in the poset of proper $\triangle$-filters of $A$.

Lemma 3.2. Let $A$ be a finite non-trivial mpM-algebra and $a \in A$. Then the following conditions are equivalent:

(i) $F(\triangle a)$ is a maximal $\triangle$-filter of $A$,

(ii) $\triangle a$ is an atom of $\triangle A$.

Proof: (i) $\Rightarrow$ (ii): From the hypothesis we have that $\triangle a \neq 0$. Suppose now that there is $x \in \triangle A$ such that $0<x \leq \triangle a$. From this assertion we have that $F(\triangle a) \subseteq F(x) \neq A$. Furthermore, by Lemma 3.1, $F(x)$ is a $\triangle$-filter of $A$. But since $F(\triangle a)$ is a maximal $\triangle$ - filter of $A$ we conclude that $F(\triangle a)=F(x)$. Therefore, $\triangle a=x$.

(ii) $\Rightarrow$ (i): From Lemma 3.1 it follows that $F(\triangle a)$ is a $\triangle$-filter of $A$. Suppose now, that there is a $\triangle$-filter $T$ of $A$ such that $F(\triangle a) \subseteq T \neq A$. Taking into account that $A$ is a finite algebra, by Proposition 2.3 we have that there is $x \in A$ such that $T=F(\triangle x)$. Therefore, $0<\triangle x \leq \triangle a$, but since $\triangle a$ is an atom of $\triangle A$ we conclude that $\triangle a=\triangle x$. These statements imply that $F(\triangle a)$ is a maximal $\triangle$-filter of $A$.

From now on, if $X$ is a non-empty subset of $A$ we will denote by $|X|$ the cardinal number of $X$. Furthermore, if $A, B \in \boldsymbol{m} \boldsymbol{p} \boldsymbol{M}$, we will write $A \simeq B$ if there is an isomorphism from $A$ to $B$.

Next corollary is a direct consequence of Lemma 3.2.

Corollary 3.3. Let $A$ be a non-trivial finite mpM-algebra. Then, $|\mathcal{M}(A)|=|\mathcal{A} t(\triangle(A))|$, where $\mathcal{M}(A)$ denotes the set of all maximal of $\triangle$ filters of $A$.

REMARK 3.4. Bearing in mind a well-known result of A. Monteiro ([15]), from (W1), (W4), (W6) and (W9), we conclude that all proper $\triangle$-filter of an mpM-algebra $A$ is the intersection of maximal $\triangle$-filters of $A$. This assertion implies that $\{1\}$ is the intersection of all maximal $\triangle$-filters of $A$. 
Theorem 3.5 will give us the announced factorization of the finite $m p M$ algebras.

Theorem 3.5. Let $A$ be a non-trivial finite mpM-algebra. Then $A \simeq$ $\prod_{i=1}^{n} A / F\left(\triangle a_{i}\right)$, where $\left\{\triangle a_{i}\right\}_{1 \leq i \leq n}$ is the set of all the atoms of $\triangle A$.

Proof: Let $h: A \longrightarrow \prod_{i=1}^{n} A / F\left(\triangle a_{i}\right)$ be the function defined by $h(x)=$ $\left(q_{1}(x), \cdots, q_{k}(x)\right)$, where $q_{i}: A \rightarrow A / F\left(\triangle a_{i}\right)$ is the natural epimorphism for each $i, 1 \leq i \leq n$. From Lemma 3.2, Proposition 2.8 and Remark 3.4 we infer that $h$ is a monomorphism. Hence, it only remains to prove that $h$ is onto. Let $y=\left(y_{1}, \ldots, y_{n}\right) \in \prod_{i=1}^{n} A / F\left(\triangle a_{i}\right)$. Since $q_{i}$ is onto for each $i, 1 \leq$ $i \leq n$, we have that there is $x_{i} \in A$ such that $q_{i}\left(x_{i}\right)=y_{i}$. Let us consider $x=\bigvee_{i=1}^{n}\left(x_{i} \wedge \triangle a_{i}\right)$. Furthermore, taking into account Proposition 2.5 and the fact that $q_{j}\left(\triangle a_{i}\right) \in \triangle\left(A / F\left(\triangle a_{i}\right)\right)$, we conclude that $q_{j}\left(\triangle a_{i}\right) \in\{\overline{0}, \overline{1}\}$. If $q_{j}\left(\triangle a_{i}\right)=\overline{1}$, whenever $j \neq i$, then $\triangle a_{i} \in F\left(\triangle a_{j}\right)$ and so, $\triangle a_{j}<\triangle a_{i}$, which contradicts the fact that $\triangle a_{i}$ is an atom of $\triangle A$. Thus, $q_{j}\left(\triangle a_{i}\right)=\overline{0}$ for all $j \neq i$ and $q_{i}\left(\triangle a_{i}\right)=\overline{1}$. From these assertions we conclude that $\left.q_{j}(x)=\bigvee_{i=1}^{n}\left(q_{j}\left(x_{i}\right)\right) \wedge q_{j}\left(\triangle a_{i}\right)\right)=q_{j}\left(x_{j}\right)=y_{j}$ for all $j, 1 \leq j \leq n$ and so, $h(x)=y$. This completes the proof.

Proposition 3.6. Let $A$ be a finite mpM-algebra, $b \in A$, and $[0, b]=\{x \in$ $A: 0 \leq x \leq b\}$. If $b \in \triangle A, b \neq 0$, then it hold:

(i) $\langle[0, b], \wedge, \vee,-], 0, b$,$\rangle is an m p M$-algebra, where $-x=\sim x \wedge b$ and \rceil $x=x^{*} \wedge b$, for all $x \in[0, b]$

(ii) $L / F(b) \simeq[0, b]$.

PROOF: (i) It is straightforward.

(ii) Let $h_{b}: A / F(b) \longrightarrow[0, b]$ be defined by $h_{b}(\bar{x})=x \wedge b$. Hence, $h_{b}$ is a well-defined bijection. Furthermore, $h_{b}$ is an $m p M$-homomorphism. Indeed, taking into account that $b \in \triangle A$ is a Boolean element, we have that $-h_{b}(\bar{x})=-(x \wedge b)=\sim(x \wedge b) \wedge b=(\sim x \wedge b) \vee(\sim b \wedge b)=\sim x \wedge b=h_{b}(\sim \bar{x})$. On the other hand, from (m11) we infer that $\rceil h(\bar{x})=(x \wedge b)^{*} \wedge b=x^{*} \wedge b=$ $h\left(\bar{x}^{*}\right)$. The proof of the remaining properties is routine. 
Theorem 3.5 and Proposition 3.6 imply the following useful result.

Corollary 3.7. Let $A$ be a non-trivial finite mpM-algebra. If $\left\{\triangle a_{i}\right\}_{1 \leq i \leq n}$ is the set of all the atoms of $\triangle A$, then $A \simeq \prod_{i=1}^{n}\left[0, \triangle a_{i}\right]$.

\section{Free $m p M$-algebras}

In this section, which is the core of this paper our aim is focused on determine the structure of the free finitely generated $m p M$-algebras and a formula to calculate its cardinal number in terms of the number of the free generators.

In what follows, we will denoted by $\mathcal{L}(c)$ the free $m p M$-algebra with a set $G$ of free generators such that $|G|=c$, where $c$ is a cardinal number, $0<c<\omega$. The notion of free $m p M$-algebras is defined in the usual way and since $m p M$-algebras are equationally definable, for any cardinal number $c$, $c>0$, the free algebra $\mathcal{L}(c)$ exists and it is unique up to isomorphism ([4]). Bearing in mind (TM2), (TM3) and well-known results of universal algebra we conclude that

$$
\text { (I) } \mathcal{L}(n)=A_{1}{ }^{\left|\mathcal{M}_{1}\right|} \times A_{2}{ }^{\left|\mathcal{M}_{2}\right|} \times A_{3}{ }^{\left|\mathcal{M}_{3}\right|}
$$

where $A_{i}, 1 \leq i \leq 3$, are the non-isomorphic simple $m p M$-algebras, $\mathcal{M}_{j}=$ $\left\{M \in \mathcal{M}(\mathcal{L}(n)): \mathcal{L}(n) / M \simeq A_{j}\right\}$, and $\mathcal{M}(\mathcal{L}(n))$ is the set of all maximal deductive systems of $\mathcal{L}(n)$.

Let $A, A^{\prime} \in \boldsymbol{m} \boldsymbol{p} \boldsymbol{M}$. From now on we will denote by $\operatorname{Epi}\left(A, A^{\prime}\right)$ and Aut $(A)$ the set of all epimorphisms from $A$ to $A^{\prime}$ and the set of all automorphisms of $A$, respectively.

Next, we are going to compute $\left|\mathcal{M}_{j}\right|, 1 \leq j \leq 3$. Thus, we have

Lemma 4.1. $\left|\mathcal{M}_{j}\right|=\frac{\left|\operatorname{Epi}\left(\mathcal{L}(n), A_{j}\right)\right|}{\left|A u t\left(A_{j}\right)\right|}, 1 \leq j \leq 3$.

Proof: Let $\alpha: \operatorname{Epi}\left(\mathcal{L}(n), A_{j}\right) \longrightarrow \mathcal{M}_{j}$ be the function defined by $\alpha(h)=$ $\operatorname{ker}(h)$ [4, Definition 6.7]. Hence, $\alpha$ is onto. Indeed, for each $M \in \mathcal{M}_{j}$ suppose that $f=\gamma_{M} \circ q_{M}$, where $q_{M}$ is the natural map and $\gamma_{M}$ is the $m p M$-isomorphism from $\mathcal{L}(n) / M$ in $A_{j}$. Thus, $f \in \operatorname{Epi}\left(\mathcal{L}(n), A_{j}\right)$ and $\operatorname{ker}(f)=M$. Consequently $\alpha(f)=M$. Furthermore, if $M \in M_{j}$ and 
$\alpha(h)=M$, then $\alpha^{-1}(M)=\left\{g \circ h: g \in A u t\left(A_{j}\right)\right\}$. Therefore, $\left|\mathcal{M}_{j}\right|$. $\left|A u t\left(A_{j}\right)\right|=\left|\operatorname{Epi}\left(\mathcal{L}(n), A_{j}\right)\right|$ and thus we conclude the proof.

In what follows if $A \in \boldsymbol{m p} \boldsymbol{M}$ and $G \subseteq A$ we will denote by $[G]$ the $m p M$-subalgebra of $A$ generated by $G$. Now, let $F^{*}\left(G, A_{j}\right)=\{f: G \longrightarrow$ $\left.A_{j}:[f(G)]=A_{j}\right\}, 1 \leq j \leq 3$. Then, we have

Lemma 4.2. $\left|\operatorname{Epi}\left(\mathcal{L}(n), A_{j}\right)\right|=\left|F^{*}\left(G, A_{j}\right)\right|, 1 \leq j \leq 3$.

Proof: Let $\beta: \operatorname{Epi}\left(\mathcal{L}(n), A_{j}\right) \longrightarrow F^{*}\left(G, A_{j}\right)$ be the function defined by $\beta(h)=h / G$, where $h / G$ is the restriction of $h$ to $G$. It is simple to verify that $\beta$ is injective. Moreover, for each $f \in F^{*}\left(G, A_{j}\right)$ there is a unique homomorphism $h_{f}: \mathcal{L}(n) \longrightarrow A_{j}$ which extends $f$. Besides, $h_{f}(\mathcal{L}(n))=$ $h_{f}([G])=[f(G)]=A_{j}$. Therefore, $\beta$ is onto.

On the other hand, it is simple to verify that $\left|\operatorname{Aut}\left(A_{1}\right)\right|=\left|\operatorname{Aut}\left(A_{2}\right)\right|=$ 1. Hence, as a direct consequence of Lemma 4.1 and Lemma 4.2 we conclude

Corollary 4.3. (i) $\left|\mathcal{M}_{1}\right|=2^{n}$,

(ii) $\left|\mathcal{M}_{2}\right|=3^{n}-2^{n}$.

LeMma 4.4. $\left|\mathcal{M}_{3}\right|=2^{n-1}\left(2^{n}-1\right)$.

Proof: From Lemma 4.1, Lemma 4.2 and taking into account that $\left|A u t\left(A_{3}\right)\right|$ $=2$, we infer that

$$
\left|\mathcal{M}_{3}\right|=\frac{\left|F^{*}\left(G, A_{3}\right)\right|}{2} .
$$

Furthermore, since $A_{1}$ is the unique subalgebra of $A_{3}$ we have that $\left|F^{*}\left(G, A_{3}\right)\right|$ $=4^{n}-2^{n}=2^{n}\left(2^{n}-1\right)$. Thus, the proof is completed.

From (I) and the above results we have shown

TheOREM 4.5. Let $\mathcal{L}(n)$ be the free $m p M$-algebra with $n$ free generators. Then its cardinality is given by the following formula:

$$
|\mathcal{L}(n)|=2^{2^{n}} \times 3^{3^{n}-2^{n}} \times 4^{2^{n-1}\left(2^{n}-1\right)} .
$$

Example 4.6. By Theorem 4.5 we have that for $n=1$

$$
|\mathcal{L}(1)|=2^{2} \times 3 \times 4=48
$$

and its Hasse diagram is the one indicated in the figure: 


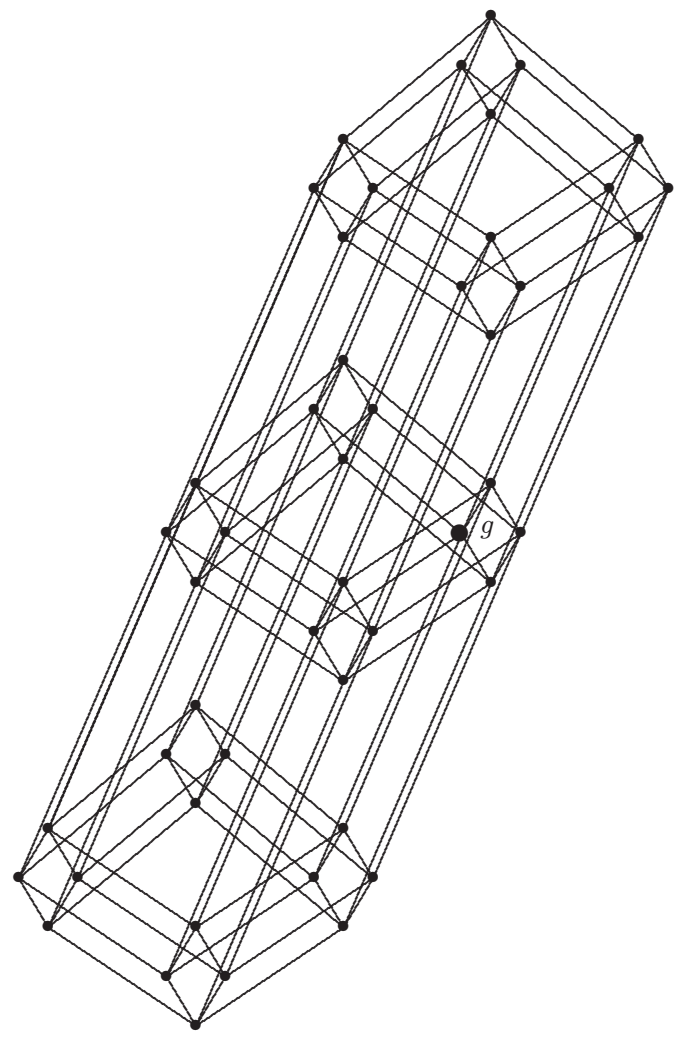

\section{Final Remark}

It is worth mentioning that, following a different reasoning that the one used in [7, Remark 3.1], the results established in Section 4 allow us to assert that $\mathbf{m p M}$ and the variety of tetravalent modal algebras do not coincide. Indeed, I. Loureiro ([14]) determined the free tetravalent modal algebra $\mathcal{T}_{4}(n)$ with $n$ free generators and she proved that its cardinal number is

- $\left|\mathcal{T}_{4}(1)\right|=2^{2^{n}} \times 3^{3^{n}-2^{n}} \times 4^{2^{n-1}\left(2^{n}+1\right)-3^{n}}$.

Hence, if $n=1,\left|\mathcal{T}_{4}(n)\right|=12$ and its Hasse diagram is the one indicated bellow: 


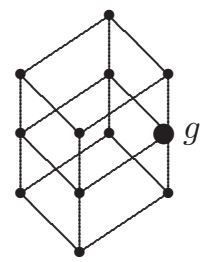

\section{References}

[1] R. Balbes, Ph. Dwinger, Distributive Lattices, University of Missouri Press, Columbia, 1974. Zbl0321.06012, MR0373985.

[2] G. Birkhoff, Lattice Theory, American Mathematical Society, Col Pub., 25 3rd ed., Providence, 1967. Zbl0153.02501, MR0227053.

[3] V. Boicescu, A. Filipoiu, G. Georgescu, S. Rudeanu, ŁukasiewiczMoisil Algebras, North-Holland Publishing Co., Amsterdam, 1991. Zbl0726.06007, MR1112790.

[4] S. Burris, H. P. Sankappanavar, A Course in Universal Algebra, Graduate Texts in Mathematics, Vol. 78, Springer-Verlag, Berlin, 1981. Zbl0478.08001, MR0648287.

[5] A. V. Figallo, Tópicos sobre álgebras modales 4-valuadas, Proceeding of the IX Simposio Latino-Americano de Lógica Matemática, (Bahía Blanca, Argentina, 1992). Notas de Lógica Matemática 39(1992), pp. 145-157. MR1332541.

[6] A. V. Figallo, P. Landini, Notes on 4-valued modal algebras, Preprints del Instituto de Ciencias Básicas, Universidad Nacional de San Juan 1(1990), pp. 28-37. Zbl0858.03062.

[7] A. V. Figallo, N, Oliva, A. Ziliani, Modal pseudocomplemented De Morgan algebras, Acta Universitatis Palackianae Olomucensis. Facultas Rerum Naturalium. Mathematica 53, 1(2014), pp. 65-79. MR3331071.

[8] J. Font, M. Rius, An abstract algebraic logic approach to tetravalent modal logics, Journal of Symbolic Logic 65(2000), pp. 481-518. Zbl1013.03075, MR1771068.

[9] V. Glivenko, Sur quelques points de la logique de M. Brouwer, Academie Royale de Belgique. Bulletin de la Classe des Sciences 15 (1929), pp. 183-188. Zbl55.0030.05. 
[10] G. Grätzer, H. Lakser, The structure of pseudocomplemented distributive lattices II. Congruence extension and amalgamation, Transactions of the American Mathematical Society 156 (1971), pp. 343-358. Zbl0244.06011, MR0274359.

[11] T. Hecht, T. Katrin̆ák, Principal congruences of p-algebras and double palgebras, Proceedings of the American Mathematical Society 58 (1976), pp. 25-31. Zbl0352.06006, MR0409293.

[12] J. Kalman, Lattices with involution, Transactions of the American Mathematical Society 87 (1958), pp. 485-491. Zbl0228.06003, MR0095135.

[13] I. Loureiro, Axiomatisation et propriétés des algèbres modales tétravalentes, Comptes Rendus Mathematique de l'Académie des Sciences Paris 295 (1982), Série I, pp. 555-557. Zbl0516.03010, MR0685023.

[14] I. Loureiro, Algebras Modais Tetravalentes, PhD thesis, Faculdade de Ciências de Lisboa, Lisboa, Portugal, 1983.

[15] A. Monteiro, La sémisimplicité des algèbres de Boole topologiques et les systémes déductifs, Revista de la Unión Matemática Argentina 25 (1975), pp. 417-448.

[16] Yu. M. Movsisyan, V. A. Aslanyan, Boole-De Morgan Algebras and QuasiDe Morgan Functions, Communications in Algebra 42 (2014), pp. 4757477, Zbl 1338.06010, MR3210411.

[17] Yu. M. Movsisyan, V. A. Aslanyan, Super-De Morgan Functions and Free De Morgan Quasilattices, Central European Journal of Mathematics 12 (2014), pp. 1749-1761. Zbl 1346.08004, MR3232637.

[18] Yu. M. Movsisyan, V. A. Aslanyan, De Morgan Functions and Free De Morgan Algebras, Demonstratio Mathematica 2 (2014), pp. 271-283.

[19] P. Ribenboim, Characterization of the sup-complement in a distributive lattice with last element, Surma Brasil Mathematics 2 (1949), pp. 4349. Zbl0040.01003, MR0030931.

[20] A. Romanowska, Subdirectly irreducible pseudocomplemented De Morgan algebras, Algebra Universalis 12 (1981), pp. 70-75. Zbl0457.06009, MR0608649.

[21] H. Sankappanavar, Pseudocomplemented Okham and Demorgan algebras, Zeitschrift für mathematische Logik und Grundlagen der Mathematik 32 (1986), pp. 385-394. Zbl0612.06009, MR0860024.

[22] H. Sankappanavar, Principal congruences of pseudocomplemented Demorgan algebras, Zeitschrift für mathematische Logik und Grundlagen der Mathematik 33 (1987), pp. 3-11. Zbl0624.06016, MR0885477. 
[23] J. Varlet, Algèbres de Eukasiewicz trivalentes, Bulletin de la Société Royale des Sciences de Liège, (1968), pp. 9-10. Zbl0175.26604

\author{
Aldo V. Figallo and Nora Oliva \\ Instituto de Ciencias Básicas \\ Universidad Nacional de San Juan \\ (5400) San Juan, Argentina \\ Alicia Ziliani \\ Instituto de Matemática \\ Universidad Nacional del Sur \\ (8000) Bahía Blanca, Argentina \\ e-mail: aziliani@gmail.com
}

e-mail: avfigallo@gmail.com, nora.oliva1@gmail.com 